\title{
Teaching undergraduates about familial breast cancer: comparison of a computer assisted learning (CAL) package with a traditional tutorial approach
}

\author{
Zosia Miedzybrodzka*,1,2, Neil M Hamilton ${ }^{3}$, Helen Gregory ${ }^{4}$, Benedict Milner ${ }^{2}$, Iciar Frade ${ }^{3}$, \\ Tracy Sinclair ${ }^{3}$, Jill Mollison $^{5}$ and Neva Haites ${ }^{1,2}$
}

${ }^{1}$ Department of Medicine \& Therapeutics, University of Aberdeen, Aberdeen, UK; ${ }^{2}$ Department of Molecular \& Cell Biology, University of Aberdeen, Aberdeen, UK; ${ }^{3}$ Faculty of Medicine \& Medical Sciences CAL Unit, University of Aberdeen, Aberdeen, UK; ${ }^{4}$ Grampian University Hospitals NHS Trust, Department of Medical Genetics, Aberdeen, UK; ${ }^{5}$ Department of Public Health, University of Aberdeen, Aberdeen, UK

We have developed a computer assisted learning package for teaching clinical medical students about familial breast cancer. It explains the principles of genetic predisposition to breast cancer, the association with other cancers, the principles of family history taking and confirmation, risk assessment and possible interventions. Clinical medical students were randomised to either conventional teaching or CAL, 48 students attended the evaluation session. Students randomised to conventional teaching received a 20 min mini-lecture, those randomised to CAL completed the package with technical, but not academic support available. At the end of the intervention both groups of students completed a short written assessment of acceptability and knowledge and understanding of breast cancer genetics. There was no significant difference between the CAL and mini-lecture groups in terms of marks or acceptability. Thus CAL appears to be an acceptable and effective method of teaching clinical medical students about familial breast cancer. Although time consuming to develop, CAL can be used in a variety of settings to increase curriculum flexibility. Methods of motivating students to complete the CAL, and of providing educational support are being explored.

European Journal of Human Genetics (2001) 9, 953-956.

Keywords: breast cancer; genetics; CAL; teaching; evaluation; C\&IT

\section{Introduction}

As services for management of familial breast cancer become established, it is important for medical students to have some understanding of disease mechanisms and management options. In recent years, computer assisted learning (CAL) has been increasingly used in undergraduate medical education. CAL allows students to work at their own pace, encourages problem solving and independent learning and can reduce didactic teaching. ${ }^{1}$

${ }^{*}$ Correspondence: Dr Z Miedzybrodzka, Medical Genetics, Medical School, Foresterhill, Aberdeen AB25 2ZD, UK. Tel: 01224552120 ; Fax: 01224 559390; E-mail: zosia@abdn.ac.uk

Received 7 August 2001; revised 24 October 2001; accepted 30 October 2001
We have developed a CAL application (termed here as a Model Patient) for teaching clinical medical students about familial breast cancer. We are not aware of any other CAL application designed for medical student use in this area. We describe a comparative evaluation of the acceptability and effectiveness of CAL compared with traditional methods for teaching students about familial breast cancer and its management.

Materials and Methods

CAL application development

The CAL application as developed by a team comprising clinical geneticists and specialists in CAL technology. The aim of the material was to explain the underlying principles 
(10) Computer assisted learning in familial breast cancer

954

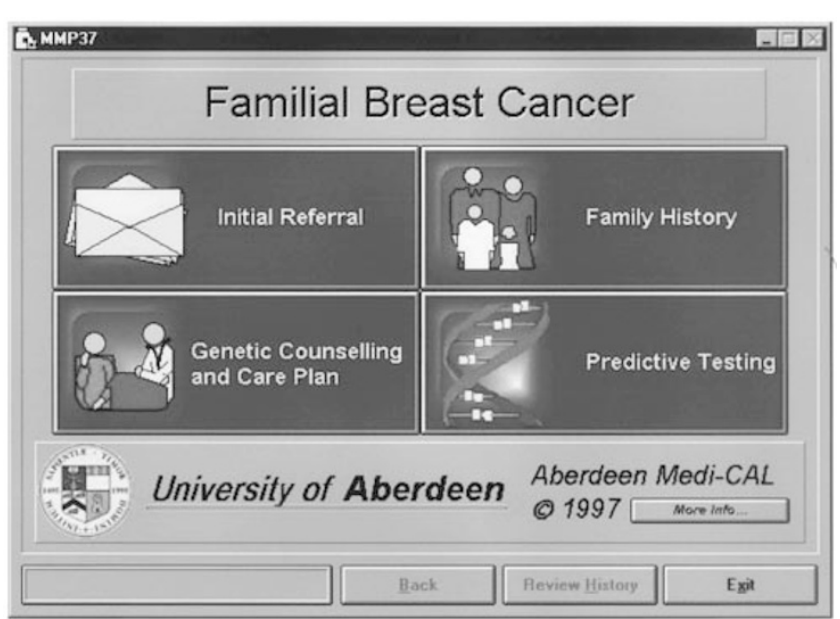

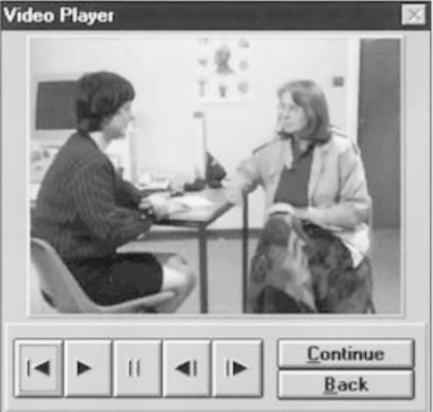

(b) Model patient discussing family history of breast cancer with general practitioner (video)

(a) First screen

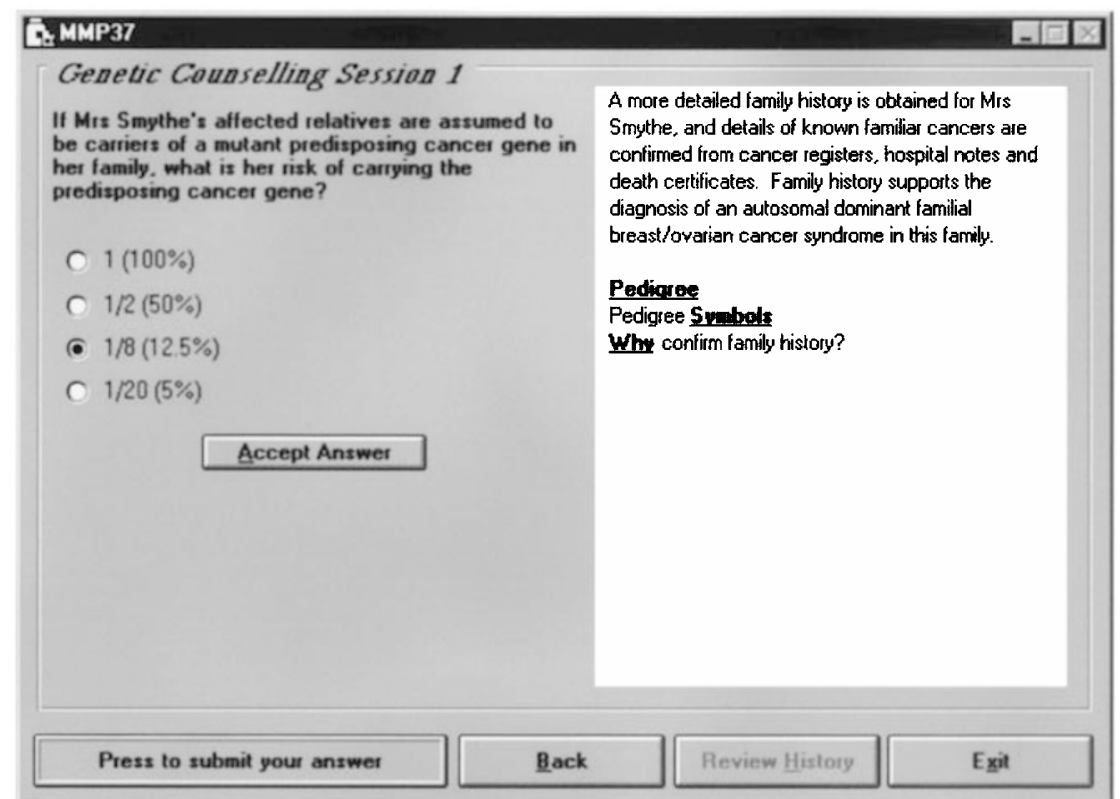

(c) Example of interactive "question" screen

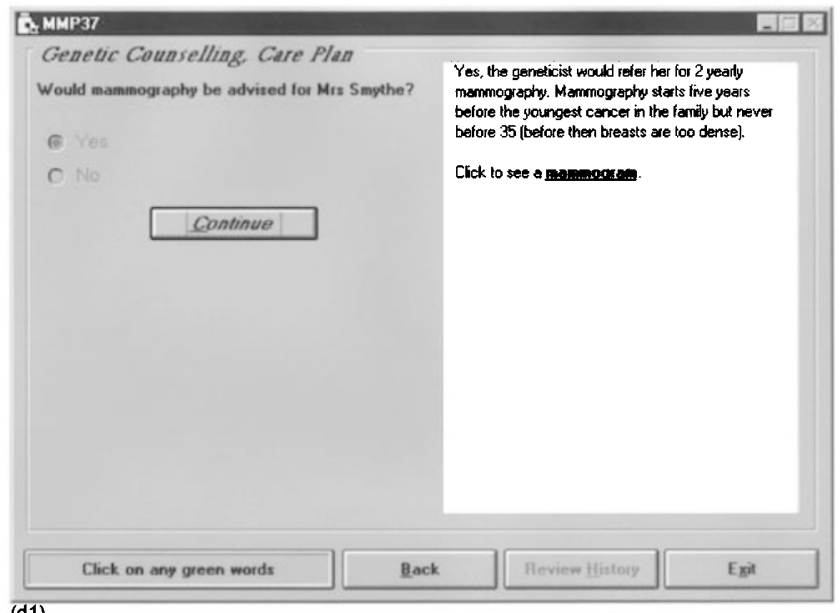

(d1)

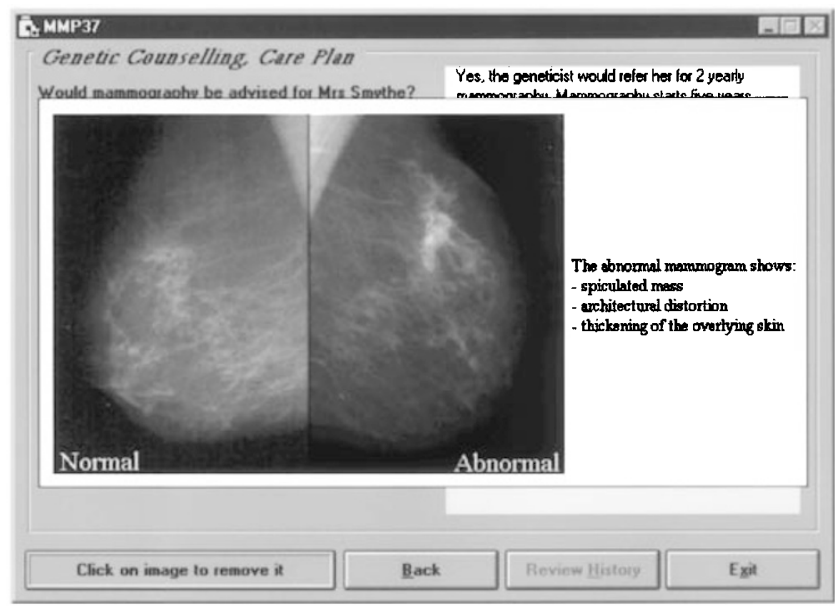

(d 1 \& 2) Interactive mammography question and example

Figure 1 Examples of screen shots from CAL application. 
of genetic predisposition to breast cancer and its management. The objectives were to familiarise students with autosomal dominant inheritance of a cancer predisposing gene, the two-hit hypothesis, association of breast cancer with other cancers, the principles of family history taking, pedigree drawing and confirmation risk estimation and management strategies for those at risk and the place of mutation testing.

The application used an interactive 'Model Patient' approach $^{1}$ in which the student is led through an at-risk patient's process of care from presentation to the general practitioner to consultations at the genetic clinic and screening options available. Examples of 'screens' used by the student are shown in Figure 1. The interactive multimedia CAL package was developed using Microsoft Visual BASIC Pro and incorporated AVI digital video.

Innovative programming allowed us to author the Multilevel Model Patients within a one month period using a specially developed 'engine'. This engine was not a template - a programmer was still required to develop the CAL, and the application could be tailored to our exact requirements. The amount of time invested by the subject experts in the CAL development process was estimated to be $40 \mathrm{~h}$.

\section{Subjects}

All the 171 fourth year clinical medical students from Aberdeen University were randomised to two groups prior to the evaluation. Students had had one previous lecture about cancer genetics one year earlier. Randomisation was stratified by gender and academic achievement, based on final mark for year three (above average, average, and below average).

\section{Interventions}

Those students randomised to CAL completed the CAL package with technical, but not academic support available. The time taken for completion of the package was logged. Students randomised to conventional teaching participated in a structured 20 min mini-lecture (with opportunities for questions) led by a clinician (ZM, HG). The aims and objectives for both modes of educational intervention were the same, and both clinicians used the same audio-visual aids for the tutorials.

\section{Assessment}

At the end of the intervention both groups of students completed a short written assessment comprising:

1. Likert scales asking students to rate how enjoyable, useful, interesting and well explained the subject matter was, and ease of use of the computing software and hardware.

2. Modified essays and multiple choice questions assessing knowledge and understanding of breast cancer genetics.

\section{Results}

Of the 55 students randomised to CAL, 16 (29\%) attended the session, and of the 116 randomised to receive a tutorial, 32 (28\%) attended. There was no difference in academic achievement between attenders and non-attenders nor between randomised groups in those attending. There was a greater proportion of males who attended (51 versus 39\%) and in the CAL group (63 as opposed to $45 \%$ in the tutorial group) although these differences did not achieve statistical significance. The mean time taken by students to complete the CAL application was $16.4 \mathrm{~min}$ (SD 4.4), the range was 8 to $23 \mathrm{~min}$.

There was no significant difference between the CAL and mini-lecture groups in terms of marks or acceptability (see Table 1 ). Numbers are small for secondary analysis of data. Time taken to complete the CAL application varied

Table 1 Assessment marks (out of 42) by mode of teaching and Likert scale scores for acceptability of the session by teaching method.

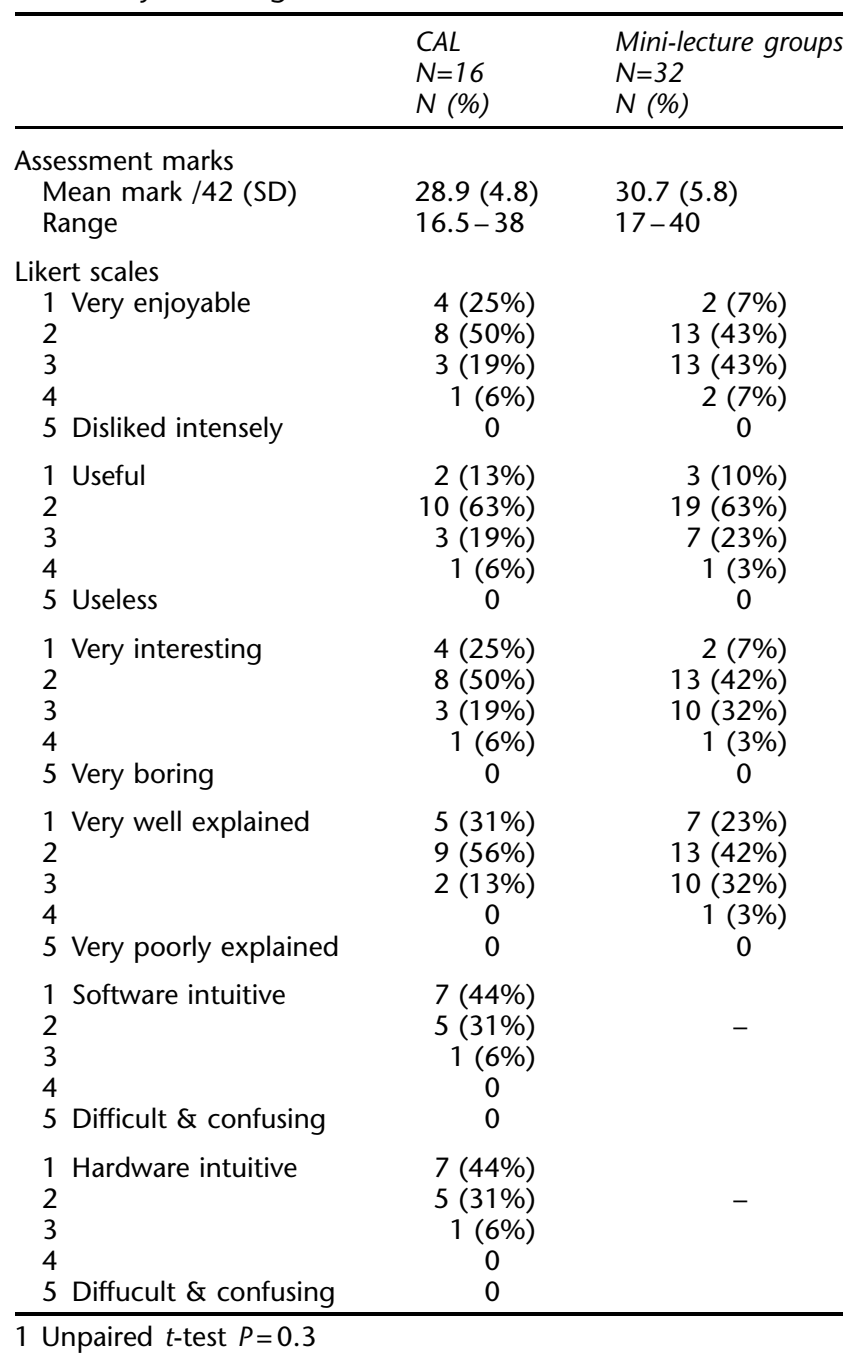


widely between students, but did not appear to influence marks.

\section{Discussion}

We acknowledge the limitations of our study, primarily the small sample size due to the low attendance rate. However, our randomised design did attempt to minimise bias and we believe that our study has indicated that CAL is acceptable and appears to be an effective method of teaching clinical medical students about familial breast cancer. Further evaluations are required to confirm these findings. The CAL package can be run across suitable networks or on stand alone PCs. A second version of the application that can be run across the World Wide Web has recently been produced. ${ }^{2}$ Using this new modular development approach we have reduced development times further and increased the flexibility of the application. Although relatively time consuming to develop compared to a standard lecture, the CAL application could be used in a variety of settings to increase curriculum flexibility. For example, if used in selflearning, clinical staff need not be in attendance. Methods of motivating students to complete the application are being explored, for example using electronic logging of accredita- tion on completion. Likewise use of information technology mediated curriculum support, such as e-mail, discussion lists or desk-top video contact with lecturers, are being piloted and might be used in conjunction with this package. Future work is planned to investigate further inter-student differences in achievement with different teaching methods. This approach may also prove useful in educating other groups about familial breast cancer.

The CAL application is available by contacting NM Hamilton, e-mail: mil001@abdn.ac.uk

\section{Acknowledgements}

This research and CAL development was supported by the University of Aberdeen, BM was funded by European Commission (Demonstration Project No. BMH4-CT96-1133).

\section{References}

1 Hamilton NM. Furnace J, Duguid KP, Helms PJ, Simpson JG: Development and integration of CAL: a case study in medicine. Medical Education 1999; 33: 298-305.

2 Robinson DA, Lester CR, Hamilton NM: Delivering Computer Assisted Learning across the WWW. Computer Networks and ISDN Systems 1998; 30: 301-307. 